INTERNATIONAL DESIGN CONFERENCE - DESIGN 2018

https://doi.org/10.21278/idc.2018.0357

\title{
EXPLORING NEW FUNCTIONALITIES IN CULTURAL HERITAGE SPACES - DESIGNING DIFFERENT MUSEUM TRAILS WITH LOW COST TECHNOLOGIES
}

\author{
S. Khan, S. Rosa and C. Germak
}

\begin{abstract}
Different museums and cultural heritage institutions are seeking new ways to improve access to their artefacts and collections. The huge impact of the digital revolution shed some light on diverse approaches to minimize the visitor's museum experience disruption and enhance the accessibility to the exhibits. This paper describes the design of a sensor system by improving the access to the cultural resources adopting a non invasive and low cost technology. The study will provide new suggestions to analyze visitors' behaviour and their needs and design new visiting trails for the museum.
\end{abstract}

Keywords: interaction design, user centred design, user experience, cultural heritage, visitors studies

\section{Introduction}

In the last two decades museum experience has slowly acquired more importance in the applied visitor and design studies field (Falk and Dierking, 2016). Since the museum experience is becoming more and more important, researchers and scholars have started to investigate new ways of engaging more people with cultural heritage resources and avoiding issues that can disrupt the museum visit such as museum fatigue and hypercongestion can be crucial. In addition, investigating different research methods and taking into account human, socio-cultural factors and last but not least, the raise of interaction technologies (Falk and Dierking, 2016) have given new hints on how to enhance the museum's inclusivity. Studies over the past two decades have provided important information on how the global impact of the digital revolution is influencing the museum and visitor studies. According to Partarakis et al. (2016): "The global impact of the digital revolution in the cultural sector worldwide brings about the need to ensure the accessibility of physical exhibits', interactive digital exhibits, digital media and digital content for disabled people." This paper addresses the accessibility of cultural heritage resources, and the need for a new approach to accessible user interaction with cultural heritage exhibits.

Physical and cognitive issues related to the museum experience started to be increasingly relevant because of the change of paradigm of the museum as an institution. Therefore, museums have gradually shifted their main educational role to a leisure oriented duty: they have become a place to experience the collections where it's possible to increase the personal knowledge by a specific exhibit's storytelling. As a result of the museum's social role shift, this cultural institution started to appeal to a more varied type of visitor, ranging from connoisseurs of the collection to those who face the artefacts for the first time.

And according to (Bollo and Dal Pozzolo, 2005), the less the visitors know a priori about the museum collection, the more their behaviour and learning experience can be disrupted by the spatial settings of 
the exhibits. Hence, the visitors can explore the collections freely, but they can also ignore specific objects or showcases inside the space, neglecting disclosure from the narration proposed by the curators. Furthermore, visitors often ignore the reasons that lead the museum's professionals to chose a specific arrangement for the exhibit, and why specific objects are shown in the display since the public often ignore the curator taxonomy and the selection criteria used to give a hierarchy to the artefacts (Screven, 1976). For this reason, it's important to focus on how to enhance the museum signage, test its efficiency and narrative power, and where possible, strengthen it with some adjustments with low cost technology (Pugacs et al., 2017).

We used a qualitative case study approach to investigate the applicability of a low cost technology implementation for the design's exhibit, and how it can effectively enhance the museum experience. Furthermore, in order to design and explore different solutions related to the museum experience's issues we conducted a preliminary workshop with the museum's staff and distributed questionnaires to the visitors as a part of our case study.

In order to speculate the functionalities of a low cost technology for the design's exhibit, a team was formed to tackle the issues related to the case study. This team was composed of professionals from museology, psychology, engineering, and design. The research team was set up to identify and solve issues that the visitors were having with their museum experience; this was achieved by the application of low cost technology.

The case study presented in this paper is an ongoing project that aims to develop a sensor system that can help to enhance the visitor's museum experience. The sensor system was designed to support the use of low cost proximity sensors by providing minor adjustments to the existing display, answering the needs emerged from a workshop with the project's stakeholders.

The aim of the project is to develop new exhibits paths supplied with the help of low cost and impact technology devices. In order to investigate the opportunity to use technological solutions to tackle the issue, it was essential to proceed first with a workshop with the staff of the museum where the case study took place, and to schedule all the steps of the project. Secondly, it was crucial to obtain a definite mapping of the visitor's behavioural paths, in order to tackle the issues that can affect and disrupt the museum visit, (mostly stress, museum fatigue and hypercongestion).

This paper attempts to show that is possible to enhance the visit by providing the museum visitors a tool to navigate inside the museum. The design approach we use aims to design and give guidelines to implement the user's experience by giving suggestions on how to navigate inside a museum space, but at the same time, how to map and track visitor behaviour prior to a personalized path planning. By improving the museum experience, we will address the need of a shorter time length visit and also propose a different theme of interest for every new visiting trail. In addition, the improvement of the museum experience will bring benefit on the visitors' behavioural pattern, that will include less fatigue and hypercongestion by reducing the overcrowding of certain areas and diminishing the average visiting time.

This paper is divided into four parts. The first part deals with the description of the related work and the state of the art. The second part depicts the adopted approach and describes the research project. The third part points out the findings that lead to the design of the sensors system. And finally, the last part focuses on the discussion and future work.

\section{Related work}

Central to the entire discipline of visitor studies is the concept of museum experience. This experience "begins before the visit to the museum, includes experiences within the museum (interactions with staff and members of one's own group, as well as with other visitors, exhibitions, interpretive materials, and programs), and continues long after the person leaves the museum" (Falk and Dierking, 2016). Furthermore, the museum experience is something that can be designed and enhanced (Falk and Dierking, 2016) with the help of technology (Ardissono et al., 2012) in order to let the visitor learn from the artefacts.

According to King and Lord (2015) "museum learning is a voluntary, informal activity. The voluntary nature of the experience has much to do with deciding what to see within the museum space. Navigation is agency; people decide with their feet. The initial requirement for successful museum learning, whether 
online or onsite, is clear navigation." Museums often have to respond to visitors' basic needs of locating themselves into an unknown space. These needs can span from finding the toilet or the café shop to specifically looking for a showcase or the newest exhibition (Falk and Dierking, 2016), and for this reason, a tool to navigate inside the museum's space should fit the users needs with a high level of personalisation (Ardissono et al., 2012). A good level of personalisation can enhance the museum experience by tackling the physical and cognitive issues that could disrupt the visit, such as hypercongestion and museum fatigue. In addition, the museum visitors can belong to different target and age range, for this reason it can be helpful to adapt different features and contents for every user profile (Rubino et al., 2013).

\subsection{Museum experience issues and user experience insights}

The museum experience is mostly learning oriented and the leisure factor plays an important role in terms of learning (Wittlin, 1970). To allow visitors to enjoy leisure time inside the museum it's crucial to understand how people interact with the collections and the showcases. The 'stress as a human factor' can influence the perception of the museums' artefacts and could be detrimental to the learning experience that these cultural institutions usually offer. The stress variable can be composed of two main issues: hypercongestion and museum fatigue. These two issues related to visitor studies have received considerable critical attention in recent times (Falk and Dierking, 2016).

\subsection{Wayfingding, navigation and path planning}

According to Jansen (2008) space related issues concerning the museum's physical environment can be detrimental to the museum experience and the learning activity. The digital museum experience can be helpful as a tool to aggregate people with social activities and engage visitor's interaction.

As explained by Jansen, physical element in the museum environment can: "contribute to the atomization of visiting groups. This atomization disrupts visitors' abilities to engage in joint activity and to sustain interaction over time and space. In the absence of such coordinated activity and interaction, perspectival reciprocity can no longer be taken for granted - issues related to the lack of connectivity - physical space issues"

With the aim of deepening the understanding of spatial navigability in museums, a significant analysis and discussion on route planning Apps was presented by Pugacs et al. (2017).

In their detailed examination of a framework to evaluate the interactive route planning Apps in tourism, they showed that use a planning App can provide a chance to let the tourist engage with their personal will and time constraints.

Drawing on an extensive range of sources, and with the creation of journey maps, the authors set out the different ways in which: "an alternative approach allows us to achieve two things: it provides an interactive environment where the user can explore many possible itineraries with little effort. It provides an explanation of the properties of the tour, since the tour is automatically generated based on preferences indicated by the User" (Pugacs et al., 2017).

Using journey maps and heat maps to track the visitor's behaviour can be beneficial to understand the user's needs (Martella et al., 2016). According to Martella et al. (2016) the heat maps can show the amount of time spent in areas close to artworks and give valuable insights into visitors' behaviour to researches and museum professionals.

But why it is so important to understand how people navigate in the museum space? The need to understand visitors' movement is not new to the research area of museum studies. In 1988, Nathalie Heinich published a study showing that a ubiquitous technology that could fully support the exhibit's narrative power can be worthwhile to understand how people navigate and sense the museum space. Data from the studies of Heinich (1988) suggested that the multitude of museum's spaces should work cohesively with the aim to guide visitors in an educational leisure experience, giving the users freedom to access the culture at their pace. The visitor's pace is mostly driven by the given personal time they can dedicate to the visit and their personal interests. Following this purpose, the project is motivated by the pursuit of a ubiquitous exhibit technology, that can detect visitors' needs but also break down the barriers of incomprehension toward the artefacts. 


\subsection{Low cost technological solutions - proximity sensors applications}

In order to smooth the visitor navigation inside the museum space diverse large scale European museums have been interested in the development of the new visitors' trails, or have used sensor technology to monitor and analyze their visitors' needs. The users' needs ignited an impulse to speculate new approaches in the social and museological fields. One example of these investigations is the study pursued by a famous museum, the Louvre. This museum, in partnership with the MIT Seanseable City lab, started to observe visitor clustering alongside the collection's masterpieces. This research was aiming to analyse visitors' hypercongestion and clustering through the use of a series of Bluetooth proximity sensors (Yoshimura et al., 2014).

The Louvre visitor mapping provided information on the visitors' behaviour and circulation, however this research was not taken forward to find a solution to the navigation issues along the display. Instead, the science museum of Trento MUSE, located in the north east of Italy, uses a simple Bluetooth technology to suggest to the people new visiting trails by providing simple insights on the displayed objects. The Trento's museum uses a simple pop-up interface installed on a tablet given to each visitor. This tablet works not only provides audio content, but also includes the possibility to enjoy extra multimedia contents triggered by the proximity of the Bluetooth sensors. At the MUSE, the Bluetooth technology is used for a more efficient interaction with users, while at the Louvre the same devices were used to monitor and analyse visitors' behaviour.

But according to Ardissono et al. (2012), the main challenge for an enhanced museum experience was to reach an increasing level of personalised support during the museum visit, since "cultural heritage sites have a huge amount of information to present, which must be filtered and personalized in order to enable the individual user to easily access it."

Moreover, according to Ardissono et al. (2012) and Falk (2009), since the visitor's behaviour doesn't remain consistent during the visit, it may require an ongoing adaptation to each individual and situation. This is mostly due to the composition of the museum visit experience that consists of a combination of physical, socio-cultural and identity related aspects (Falk, 2009).

"Cultural heritage has been a powerful field of application for personalization for many years and recent museum studies' research acknowledged the need for personalized, individual support" (Ardissono et al., 2012). John Falk's (2009) describes the museum experience as a combination of cognitive factors. Therefore, it's possible to say that a good level of personalisation can enhance the quality of the museum visit, since visitors "may benefit from individualized support that takes into account contextual and personal attributes with a support to an ongoing adaptation." (Ardissono et al., 2012).

This paper combines the user behavioural analysis with the visits path planning. In the case studies that have been taken into account, the analytical aspects are combined with the paths suggestions, in order to give the visitors an enhanced museum experience.

\section{Understanding the collection: A case study approach}

The project's aim is to grant an improved fruition of the museum's display, enhancing visitor museum experience. In order to investigate the combination of digital and non-technological solutions to improve the visitors' experience, it was fundamental to combine different expertise from the engineering area, the design background and the humanities.

\subsection{Case study analysis to design an enhanced museum experience}

A case study approach was adopted to conduct this exploratory study on ubiquitous technologies. To capture the complexity of the phenomenon and set up the preliminary guidelines, a workshop with the project's stakeholders was organised and coordinated by the design department.

the collaborative nature of the workshop offered clear insights into the main issues that the visitors had with the museum experience. The museum management proposed the following directions to the issues:

- To expand the museum's community, including more young adults

- To design different visitor's trails

- To boost the dissemination of the museum's events and activities aimed to increase the number of visitors 


\subsection{Methodologies and data analysis: Timing, tracking and mapping visitors' behaviour}

In this section we are going to describe the procedure for the data collection, that supported the design of the enhanced museum experience concept.

\subsubsection{Workshop findings: Key points and tools to improve the visit}

The guidelines presented at the end of Section 3.1, such as the need "to expand the museum's community, including more young adults", the necessity "to design different visitors trails" and the pursuit "to boost the dissemination of the museum's events and activities aimed to increase the number of visitors" lead the workshop discussion. As this case very clearly demonstrates, it is crucial to find better strategies to make the museum experience more engaging, through simplifying the visitors' navigation inside the collection or minimizing the hypercongestion (Loomis, 1987) The large scale museum we are describing is the Egyptian museum (Museo Egizio) of Turin, in northern Italy. This Museum has welcomed more than two million visitors in almost two years after its total renovation. The main issues expressed by the museum management were the visitors' hypercongestion along the permanent collection and showcase, and the disruptive average visiting time that usually extends over four hours. The average visitor should take approximately four hours to navigate the whole display of the museum, that is developed alongside four floors of a late XVII century building. The current extension of the main and only museum path is distributed over four storeys and it spans about ten thousand square meters. These preliminary details help to understand the stress and fatigue that most of the visitors are expressing to the museum institution, it's really hard to navigate through the entire collection without exhibiting even minor signs of tiredness (Gillman, 1916).

Furthermore, the majority of the objects treasured inside the Egyptian museum's collection are related to material culture and everyday life because the museum is an archaeological institution. Being an archaeological museum means that most of the objects displayed in the showcases could be very similar to each other, for example the average visitor can become bored if the full display contains more than forty samples of the same headrest, or a hundred tiny fragments of a wide stone decoration (Tzortzi, 2015). To optimize the learning activity inside the museum, and to tackle the museum fatigue and hypercongestion, it has been fundamental to settle up a way to convey, through a form of storytelling, the meaning and the information of the artefacts along the display (Schuman and Scott, 1987).

In addition, the museum has been completely renovated in its image and display, and because of that minor changes could be operated on the showcases and the general setup. Taking into account all the issues described above, and the state of the art concerning the existing case analysed in the related works session, it would be legitimate to think about a low cost, and minor environmental impact solution to suggest different paths of visit. In this peculiar research project, a sensor system was designed to address all these issues, and the Bluetooth technology seemed the most convenient in terms of costs and device implementations. The overall project's design should consider environmental and spatial variables in order to grasp a wider scenario of the museum's space activity. The visitors' clustering and their time of permanence in proximity of the showcases can provide better picture of how to design an interaction design tool to:

- Communicate the contents of the museum's collection in a more efficient way while testing the efficacy of the permanent display;

- Deeply understand and enhance the visitor's museum experience by tackling the weaknesses of the museum trails and reinforce the key points of the display;

- Empower the museum's services, such as the bookshop and the cafeteria, and to take account of general service management standards and accessibility;

- Understand the needs of design specific pathways for families, young visitors and old visitors, while analysing people's personal interests inside the museum space;

- Enhance the potential attraction of some artefacts along the display, underlining the most and least seen zones by the visitors along the trails;

- Decrease the museum fatigue and hypercongestion phenomena to improve the visitor experience while estimating the visitors' permanence time and aggregation areas along the trails. 


\subsubsection{Timing and tracking}

Traditionally, visitor's behaviour mapping has been assessing timing and tracking constraints to evaluate the exhibits (Loomis, 1987) (Bitgood). This approach can be fruitful to highlight the visitors' expected and intended behaviour while they are approaching the collections. The behaviour's mapping can be performed manually from the data collector or could rely on sensor technology, but nevertheless it could provide valuable information to control the process about the target collections' display, that can be used for designing future exhibitions (Yalowitz and Bronnenkant, 2009).

In addition, to make the data collection even more understandable, the spatial analysis was supported by a sensible mapping technique widely used in the urban planning field (Sepe, 2007). These two approaches were essential in order to depict the gathered data in a direct way throughout a graphical visual render of the visitors' flux.

The advantages of combining the behavioural mapping and the spatial analysis is that it is possible to create floor plan heat maps and have an immediate feedback on the visitors' navigation. This technique has also been adopted by Bollo and Dal Pozzolo (2005) to track the user's behaviour, but their potential bias in the data analysis was minimized in adopting a combined framework based on specific variables; we will discuss more in Section 3.2.3.

\subsubsection{Defining visitors' scenario manual mapping and observations}

Mapping data was gathered from multiple sessions of shadowing at various time points during three months of observations. These observations were useful in the follow up phase to help designing the functionalities of the sensor system for checking the visitors' time of permanence around the artefacts and obtain information on their clustering alongside the display. The mapping has been intended as an ethological approach and has been followed from a visitors' questionnaire since in observational studies, there is a potential for bias from the real user's behaviour that can be clarified with interviews or questionnaires (Loomis, 1987).

The ethological approach has been intended in this study as a basis to attempt to understand human behaviour (Fink and Grammer, 2002) as an adaptive response to different stimuli from a museum's space. However, these methods can highlight some restraints, but they have wide applicability in museum studies (Schuman and Scott, 1987) since they can support a sharp primary examination; particularly in our case study, because we have applied a manual analysis of the visitors' behaviour.

But which kind of variables were recorded during the case study described in this paper? The visitors behaviour mapping was based on the conceptual framework proposed by Yalowitz and Bronnenkant (2009). Since there's a huge number of variables that can be analyzed in a timing and tracking study, the ones recorded for this investigation are based on Serrell's work (1998) who described a methodology to gather data tailored on the purpose of the particular case study that can include a standardized approach to rely on. In addition, the basic guidelines used to evaluate and filter the results obtained from the mapping were given during a preliminary co-design workshop that involved the staff from the institutions who actively participated in the whole project.

The following bullet list reports the Serrell's situational variables that may affect visitors' behaviour:

- Levels of crowding

- Month or season

- Day of the week

- Time of day

- Any special events or programs going on at the museum

- Any special events or programs occurring in the exhibition

- Presence of staff, carts, or other related experiences

As a result, from the Serrell's framework elaboration, the following variables in the museum's observations were taken into account:

- How much time each person could spend alongside the display

- Identify the most populated zones on each floor (visitors clustering along the display)

- Run the observations on definite days on the calendar (festive days, week- days, special city holidays, and specific scheduled events) 
- Provide basic targets of classification of the visitors (age, interests, gender)

- Investigate the reasons that motivate the average museum visit
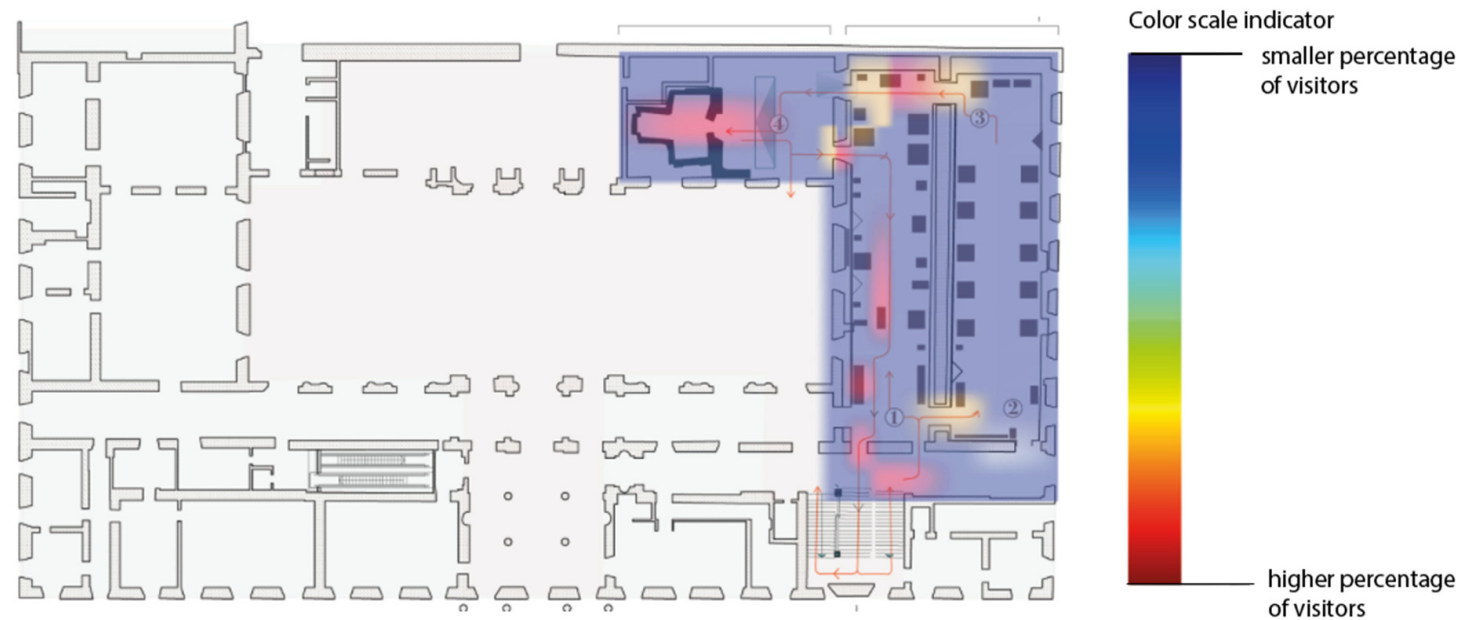

Figure 1. Visitors navigation heat map of the first floor realised after the observation sessions; The heat map depicts the clustering of people's aggregation in the museum's space; A colour indicators shift from the coldest, deep blue, to the warmest bright red;

The areas depicted in cold colours show the lower populated portions of the floor plan, while on the opposite, the warmer colour has been used to show up the more congested areas in the map

The data emerged from the observations according to the previous guidelines helped to understand the behavioural pattern of the visitors inside the museum (Gilman, 1916). The first tree variables, which define time and space constraints, have been used to develop the heat maps of the visitors preferred paths. The last two guidelines have been applied to the visitors' survey research to deepen and complete a museum experience comprehensive. To achieve this preliminary overview, it was compulsory to make an on-site examination operated by an observer. The observer has been collecting the whole data in more than 35 sessions for a period of about three months, between April and June 2016.

\section{Designing the proximity sensor system}

In order to setup an automated sensory system, we rely on low-cost common location sensors and well established techniques. In particular, for gathering information about users' location, a number of Bluetooth Low-Energy (BLE) beacons were installed inside the museum in the ground floor, located in the "Gallery of the Kings" room, around each artefact of interest. On the visitor side, a simple Android app has been developed that senses the nearest beacon, by measuring the Received Signal Strength (RSSI) from all nearby beacons, and sends this information over Wi-Fi to a central server. In our preliminary experiments, a small number of proximity sensors have been placed in the museum, and the app is running on the visitors' smartphone. By aggregating the positions of the visitors by place and time of day, we are able to generate heat maps containing the density of visitors around each artefact at any given time. This preliminary implementation of the sensor system is intended to show the feasibility of the proposed approach.

We adopted the Bluetooth technology because, according to Faragher and Harle (2014), is low-cost, and requires low maintenance efforts. In addition, this technology has been used extensively for commercial use, enabling a "new signal of opportunity with which to perform more fine-grained indoor positioning. Other indoor location methods as the Wi-Fi fingerprinting, will be less convenient to use as "Wi-Fi is a power-hungry protocol. Bluetooth Low Energy (BLE) uses the same $2.4 \mathrm{GHz} I S M$ radio band as Wi-Fi, and addresses many of these shortcomings" (Faragher and Harle, 2014)". 


\subsection{Recommending places of interest}

One important requisite is the ability to profile the visitors' preferences in order to automatically suggest personalized tours. In this work we propose the use of a recommendation engine. The idea behind recommendation engines is that users tend to like items that are similar to other items they like, and that similar people like. In a recommendation engine there are two classes of entities: users and items. The data is expressed as a so-called utility matrix, which for each user-item pair, stores the degree of preference of that user for that particular item. Values are usually integers (e.g., integers from 1 to 5 represent the number of stars that the user gave as a rating for that item). The matrix is usually sparse, since most ratings are not known. The objective is to learn embeddings that model these similarities from a set of available training data, and using these similarities to discover new and desirable things for the user.

For creating our utility matrix, we first create a list of topics of interest. Each place in the museum is manually assigned to one or more topics of interest. Then, we need to gather user ratings, which represent how much each user is interested in each topic. We use a mix of questionnaires, manual observations and automatic user location the result of questionnaires, but ideally the utility matrix ratings could be obtained by looking at how long a selected subset of test users stops near each place of interest, using the Bluetooth sensor system.

In this work we used the LightFM recommendation library (Kula, 2015), and in particular we use Weighted Approximate-Rank Pairwise loss (WARP), which works best when only positive interactions are available and the ratings are mostly sparse. In practice, WARP will randomly sample recommendations from the learned model, until it finds a pair of recommendations which it knows to be wrongly labelled, and will then only apply an update on the two wrongly labelled samples. At runtime, for each user we collect at least two ratings, then we use the learned model to predict a list of new topics.

\subsection{Recommending alternative paths}

In this study we crated heat maps, both manually and automatically generated, as a two-dimensional visual representation of data in where values are represented by colours. With these visual maps we want to exploit this information in order to suggest a tour that touches different points of interest, while minimizing hypercongestion.

We want to suggest a personal tour to the user that guarantees visiting most of the suggested places of interest, with the constraint that the tour should last less than a certain amount of time. Moreover, we want to exploit the data we have about the density of visitors around the artefacts for suggesting alternative paths to the user. The problem is formulated as a Shortest Path Problem with Time Windows (SPPTW), and it is solved using a label-setting algorithm, which is equivalent to solving a dynamic programming problem. In this work, we use the path suggestion algorithm to suggest a path be- tween consecutive places of interest, trying to avoid crowded places. The Shortest Path Problem with Time Windows is a two-resources variant of the Shortest Path Problem with resource Constraints (SPPRC) problem, in which a cost has to be minimized, while a resource needs to remain bounded between two given values for each node.

We first create an acyclic directed graph composed by a set of nodes and a set of arcs connecting adjacent nodes. A weight is associated to each arc, based on walking time between the two corresponding nodes. We want to find the shortest feasible path that satisfies the constraint on the accumulated time spent over the path. For creating the graph, we subsample the floor map; each pixel represents a node in the graph, and is assigned a visiting cost. Free areas are assigned a low cost, while obstacles are assigned a high cost. Edges are created between neighbouring pixels, with a traveling cost. Moreover, each node is assigned a time resource, based in the current density of visitors in that point. The time resource represents the amount of time lost due to a high density of people. The idea is to avoid crowded places, thus suggesting faster tours. The algorithm has been implemented using a basic implementation of the SPPTW problem included in the Boost Graph Library (Boost, n.d.). Figure 2 shows an example of the algorithm in action. The example shows a suggested path be- tween a location on top of the stairs at the bottom of the map, to a location on the top of the map. The shortest path, shown in blue, does not take into account passing through an area with a high density of visitors in the middle of the left corridor. 
The suggested path, shown in green, takes into account the heat map and, given a higher bound on the time of arrival at destination, suggests a path along the right corridor, avoiding most crowded areas.

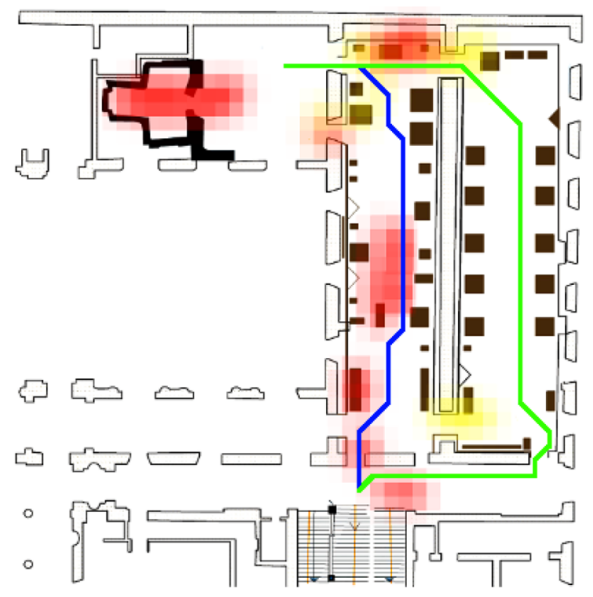

Figure 2. An example of the algorithm recommending alternative paths on a section of the ground floor; Blue line represents the shortest path; Green line shows the suggested path taking into account density heat map (yellow and red areas) while Red represents areas with a higher percentage of visitors

\section{Early results, discussion and comparisons}

The present research explores how to enhance the visitors' museum experience. The efforts made so far in this direction showed in the presented case study, that in order to achieve an enhanced museum experience it's possible to adopt low cost technology. In the analysed case study, the proximity sensor system could achieve a good level of personalisation of the user experience and minimize the effects of hypercongestion and museum fatigue to improve the user's experience. According to Ardissono et al. (2012) the visitor can be able to "exploit cultural heritage material before, during and after the visit, having different goals and requirements in each phase of their experience". So a level of preferred variables can be included to enrich the users' museum visit. On the other hand, technology can be ubiquitous and sometimes overwhelming in terms of the amount of given information, and still the content should be assisted or filtered by the curators (Ardissono et al., 2012).

As we mentioned in the introduction, a questionnaire was distributed to the 35 museum visitors to prove the need of a better museum experience. This survey has been conducted in order to:

- Understand the key issues concerning visitors' collection's fruition

- Estimate and approximate the the percentage of the visitor with orientation problem inside the museums space

- Estimate the average visit duration

- Determine the composition of the visiting groups

We can observe from the preliminary questionnaire analysis, the main issues expressed by the visitors are related to a slight tortuous path to follow in order to enjoy the permanent collection. Also the data are showing a huge average time of visit (mostly over 4 hours). Furthermore, these data have been beneficial to validate the preliminary functionalities' implementation of the sensor system, since it was possible to identify the areas and the rooms where the sensors could be located to to possibly achieve an enhanced museum experience. Also with the help of the survey was possible to have an average expected time for each visit, around four hours (Figure 3), and to understand if people could really have struggled to follow a specific narrative path. These findings helped to set up the functionalities of the proximity sensor system. From these findings, we can assume that in order to improve the museum experience it's vital to more effectively investigate the relationship between the museum and the public, and between the artefacts and the visitors. Creating an inclusive tool for understanding the artefact that 
can be driven by the creation of new visitors' paths could highlight the paucity of previous visitors' experience and enrich their visit.

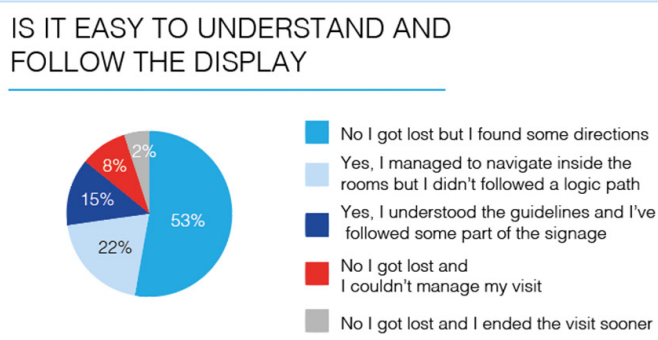

No I got lost but I found some directions Yes, I managed to navigate inside the rooms but I didn't followed a logic path

Yes, I understood the guidelines and I've followed some part of the signage

No I got lost and

I couldn't manage my visit

No I got lost and I ended the visit sooner

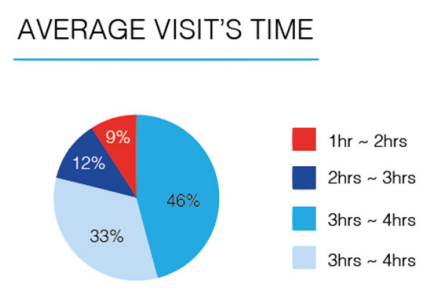

Figure 3. The visitors' survey data related to the average time devoted for a visit in the museum and the level of understanding related to the permanent collection

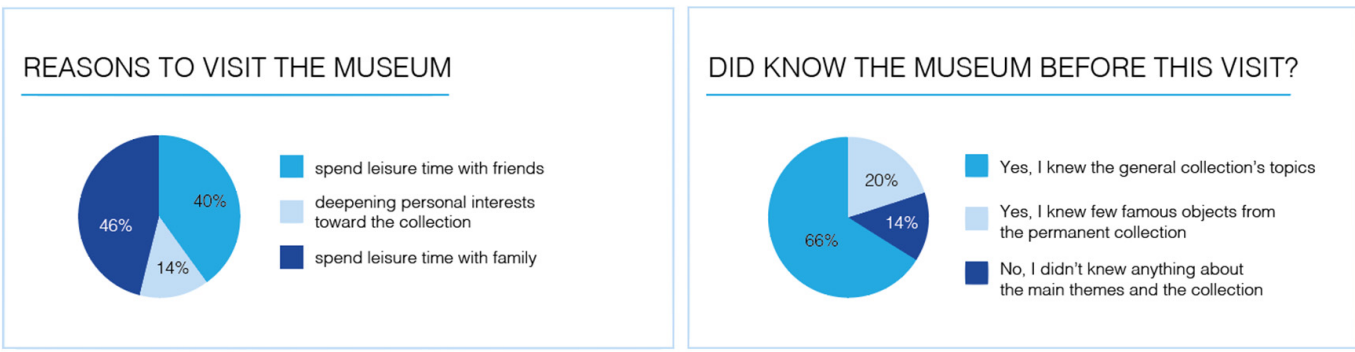

Figure 4. Visitors' survey shows some basic data about the reasons that mostly drive people to visit the museum and how knowledgeable they are about the collection

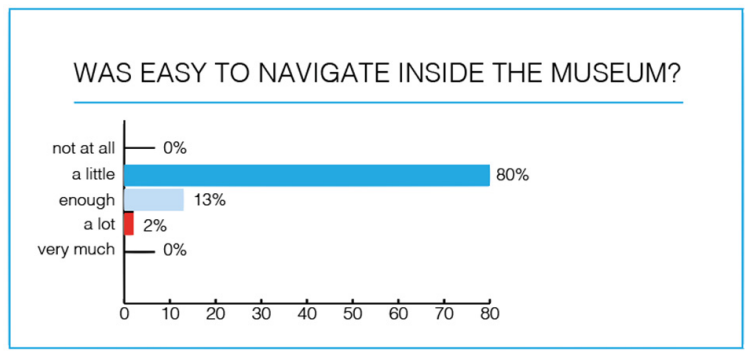

Figure 5. For most of the interviewed visitors, navigating inside the museum wasn't effortless

\section{Conclusions and future works}

The greatest challenge in this project was given by the pursuit of a technology that can join together, into a constructive dialogue, engineering, museological, architectural aspects. These aspects would be of great importance in order to broaden the museum audience and make a substantial chance of an accessible and inclusive culture. To define the future framework of activities for the next steps of this project, we analyzed the findings from Vouros (2001) and Colineau and Paris (2009). Both studies show the importance of assessing the impact of tailoring information-seeking tasks in the field of engineering management. Vouros highlights the importance of personalised content according to the user's needs while taking into account the aspects of dynamic process navigation in museum's spaces. In addition, Colineau and Paris (2009), point out the necessity of "evaluate whether providing tailored information would help people find the information they need more quickly and accurately". These frameworks support our aim of personalisation of the museum's content in order to provide to the users the information they look for and improve their understanding of the museum's collection. 
The results of this project will to show how cognitive and physical aspects can be relevant to influence any part of the museum experience, and how it's possible to achieve an enhanced museum experience with minor impact to the museum's collection and still empower its communicative content. To convey the cultural content, it's possible to adopt low cost technology and achieve a good level of interaction with the museum's visitors.

In addition, the sensor system we described in our research can be used as an interaction design tool with a double peculiarity. This tool could work both to analyse and map visitors' behaviour, and could be helpful as a tool to improve visitors' navigation along the museum's display. As we mentioned before in the related works section of this paper, other large scale museums and institutions made efforts to improve new visits and analyse visitors' clustering and behaviour, but almost never together, and not with the same technology we would like to develop and implement.

In the next steps, the sensor system will be improved by deploying more beacons, ideally one near each point of interest, in order to be able to gather punctual data about visitors' density throughout the environment. Moreover, the Android app will be improved by adding and engaging a graphical user interface for suggesting new trails to the users, based on the recommended points of interest and path given by the system. The system will operate in real time, adjusting to the density of users, in order to minimize the hypercongestion phenomenon and the stress.

In conclusion, the research aims to reach a good level of inclusion and personalisation of the museum visit, in order to define a new framework to evaluate users' satisfaction and enrichment of the museum experience.

\section{Acknowledgments}

We would like to thank the Telecom Italia JOL Crab Lab for the precious technical support and the Fondazione Museo delle Antichità Egizie in Torino for supporting this research and welcoming us in the museum's space. In addition, a special thanks goes to Veranika Lim for her invaluable support and patience.

\section{References}

Ardissono, L., Kuflik, T. and Petrelli, D. (2012), "Personalization in cultural heritage: the road travelled and the one ahead", User Modeling and User-Adapted Interaction, Vol. 22 No. 1-2, pp. 73-99. https://doi.org/10.1007/s11257-011-9104-x

Bollo, A. and Dal Pozzolo, L. (2005), "Analysis of visitor behaviour inside the museum: an empirical study", Proceedings of the 8th international conference on arts and cultural management, Vol. 2, Montreal.

Boost (n.d.), Boost c ++ libraries. [online] Boost. Available at: http://www.boost.org

Colineau, N. and Paris, C. (2009), "Does tailoring help people find the information they need?", New Review of Hypermedia and Multimedia, Vol. 15 No. 3, pp. 267-286. https://doi.org/10.1080/13614560903486151

Falk, J.H. and Dierking, L.D. (2016), The museum experience revisited, Routledge.

Faragher, R. and Harle, R. (2014), "An analysis of the accuracy of bluetooth low energy for indoor positioning applications", Proceedings of the 27th International Technical Meeting of the Satellite Division of the Institute of Navigation (ION GNSS+ 2014), September 8-12, 2014, pp. 201-210.

Fink, B. and Grammer, K. (2002), "Human ethology and evolutionary psychology. Special issue dedicated to the memory of Dr. Zdenek Klein”, Neuro Endocrinological Letters, Vol. 23 No. 4.

Heinich, N. (1988), “The Pompidou Centre and its public: the limits of a Utopian site”, In: Lumley, R. (Ed.), The Museum Time Machine: Putting Cultures on Display, pp. 199-212. https://doi.org/10.4324/9780203358825

Jansen, R. (2008), "Jurassic technology? Sustaining presumptions of intersubjectivity in a disruptive environment", Theory and Society, Vol. 37 No. 2, pp. 127-159. https://doi.org/10.1007/s11186-007-9054-9

King, B. and Lord, B. (2015), The manual of museum learning, Rowman \& Littlefield.

Kula, M. (2015), "Metadata embeddings for user and item cold-start recommendations", Proceedings of the 2nd Workshop on New Trends on Content-Based Recommender Systems co-located with 9th ACM Conference on Recommender Systems (RecSys 2015), Vienna, Austria, September 16-20, 2015, CEUR Workshop Proceedings, pp. 14-21.

Loomis, R.J. (1987), Museum visitor evaluation: New tool for management, American Association for State and Local History.

Martella, C., Miraglia, A., Frost, J., Cattani, M. and van Steen, M. (2016), "Visualizing, clustering, and predicting the behavior of museum visitors", Pervasive and Mobile Computing, Vol. 38 No. 2, pp. 430-443. https://doi.org/10.1016/j.pmcj.2016.08.011 
Pugacs, S., Helmer, S. and Zanker, M. (2017), “A Framework for Comparing Interactive Route Planning Apps in Tourism", 2nd Workshop on Engineering Computer-Human Interaction in Recommender Systems (EnCHIReS'17), Lisbon, Portugal.

Rubino, I., Xhembulla, J., Martina, A., Bottino, A. and Malnati, G. (2013), "MusA: Using Indoor Positioning and Navigation to Enhance Cultural Experiences in a Museum”, Sensors, Vol. 13 No. 12, pp. 17445-17471. https://doi.org/10.3390/s131217445

Schuman, H. and Scott, J. (1987), "Problems in the use of survey questions to measure public opinion", Science, Vol. 236 No. 4804, pp. 957-959. https://doi.org/10.1126/science.236.4804.957

Screven, C.G. (1976), "Exhibit Evaluation-A Goal-Referenced Approach", Curator: The Museum Journal, Vol. 19 No. 4, pp. 271-290. https://doi.org/10.1111/j.2151-6952.1976.tb00507.x

Serrell, B. (1998), Paying attention: Visitors and museum exhibits, American Association of Museums, Washington.

Tzortzi, K. (2015), Museum Space: Where Architecture Meets Museology, Ashgate Publishing.

Vouros, G. (2001), "Conceptual modeling of multimedia objects for user-tailored information presentations", Applied Artificial Intelligence, Vol. 15 No. 6, pp. 521-559. https://doi.org/10.1080/088395101753199560

Wittlin, A.S. (1970), Museums: In Search of a Usable Future, The MIT Press.

Yalowitz, S.S. and Bronnenkant, K. (2009), "Timing and tracking: Unlocking visitor behaviour", Visitor Studies, Vol. 12 No. 1, pp. 47-64. https://doi.org/10.1080/10645570902769134

Yoshimura, Y., Sobolevsky, S., Ratti, C., Girardin, F., Carrascal, J.P. et al. (2014), "An analysis of visitors' behavior in the louvre museum: A study using bluetooth data", Environment and Planning B: Planning and Design, Vol. 41 No. 6, pp. 1113-1131. https://doi.org/10.1068/b130047p

Sara Khan, Ph.D. candidate

Politecnico di Torino, Architecture and Design

Corso Settembrini 178, 10135 Torino, Italy

Email: s.khan17@imperial.ac.uk 